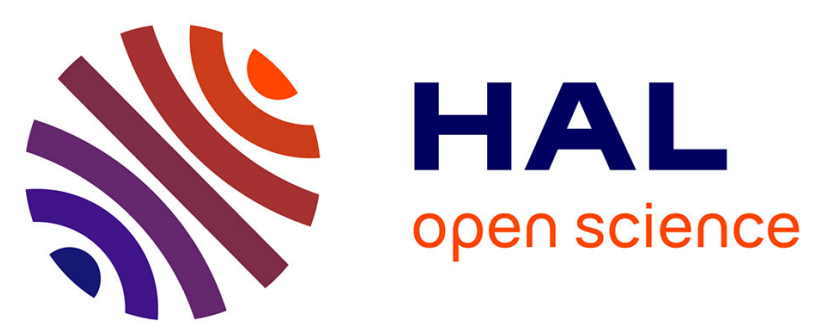

\title{
Robust image registration based on a Partition of Unity Finite Element Method
}

\author{
Oudom Somphone, Sherif Makram-Ebeid, Laurent D. Cohen
}

\section{To cite this version:}

Oudom Somphone, Sherif Makram-Ebeid, Laurent D. Cohen. Robust image registration based on a Partition of Unity Finite Element Method. 5th IEEE International Symposium on Biomedical Imaging: From Nano to Macro, 2008. ISBI 2008., May 2008, Paris, France. pp.1123-1126, 10.1109/ISBI.2008.4541198 . hal-00438776

\section{HAL Id: hal-00438776 https://hal.science/hal-00438776}

Submitted on 4 Dec 2009

HAL is a multi-disciplinary open access archive for the deposit and dissemination of scientific research documents, whether they are published or not. The documents may come from teaching and research institutions in France or abroad, or from public or private research centers.
L'archive ouverte pluridisciplinaire HAL, est destinée au dépôt et à la diffusion de documents scientifiques de niveau recherche, publiés ou non, émanant des établissements d'enseignement et de recherche français ou étrangers, des laboratoires publics ou privés. 


\title{
ROBUST IMAGE REGISTRATION BASED ON A PARTITION OF UNITY FINITE ELEMENT METHOD
}

\author{
O. Somphone ${ }^{1,2}$, S. Makram-Ebeid ${ }^{1}$ and L. D. Cohen ${ }^{2}$ \\ ${ }^{1}$ Medisys Research Lab - Philips Healthcare \\ 51, rue Carnot, B.P. 301 \\ F-92156 Suresnes, France \\ ${ }^{2}$ Université Paris-Dauphine, CEREMADE \\ Place du Maréchal de Lattre de Tassigny, \\ F-75016 Paris, France
}

\begin{abstract}
In this paper, we present a robust, hierarchical Partition of Unity Finite Element Method (PUFEM) to compute the transformation between two images, which is represented by a non-rigid, locally polynomial displacement field. The partition of unity property offers an efficient optimization scheme by breaking down the global minimization of the mismatch energy into independent, local minimizations. Moreover, the regularization introduced by our approach enables us to control the range of the smoothness. Our method was applied to cardiac ultrasound image sequences to propagate the segmentation of anatomical structures of interest.
\end{abstract}

Index Terms - Image registration, partition of unity, optical flow, robust estimation, cardiac ultrasound

\section{INTRODUCTION}

Image registration is an essential task in medical image processing, and many subsequent processes depend on it, such as segmentation propagation, strain quantification or background subtraction. Parametric representations such as Radial Basis Functions [1], B-splines [2, 3, 4] and other Finite Element Methods (FEM) [5] have been widely used for image registration. However, they use motion models with built-in smoothness that may be difficult to adapt. In [6], we introduced Partition of Unity Finite Element Method (PUFEM) for the first time, with encouraging results on synthetically warped images. The main advantage of this method compared to the aforementioned ones is the controllability of the range of the smoothness. Our current contributions include a generalization of the PUFEM for registration, through the introduction of a robust estimator in the matching term in order to reduce the influence of outliers. The paper is organized as follows. We first explain how the warping field is represented, then we present our variational approach to register a pair of images and our minimization strategy. Finally, we show some results on sequences of cardiac ultrasound images.

\section{REPRESENTATION OF THE WARPING FIELD}

In this first section, we give an overview on the mathematical framework of the PUFEM [7] that we use to model a vector field $\mathbf{u}$. Each component $u$ is a real-valued function defined on an open bounded domain $\Omega \subset \mathbb{R}^{d}$. The basic idea is to locally fit $u$ with $d$-dimensional polynomials and smoothly blend them afterwards to obtain a regular representation. To that end, we define a set $\mathcal{N}$ of nodes distributed over $\Omega$. A node $n$ is characterized by:

- a point $\mathbf{c}^{(n)} \in \Omega$, called center of the node $n$,

- an open bounded subdomain $\Omega^{(n)} \subset \mathbb{R}^{d}$ containing $\mathbf{c}^{(n)}$, called patch,

- an $\mathbb{R}$-valued function $\varphi^{(n)}$ defined on $\mathbb{R}^{d}$, called $P U$ function, whose support is included in $\Omega^{(n)}$,

- a set $\mathcal{B}^{(n)}=\left\{p_{r}^{(n)} \mid r \in \mathcal{R}^{(n)}\right\}$ of functions from $\Omega^{(n)}$ to $\mathbb{R}$, called the local basis at node $n$, with $\mathcal{R}^{(n)}$ being the set of indices for the local basis.

We choose the $p_{r}^{(n)}$ to be monomials of all degrees up to $q$, with $\mathbf{c}^{(n)}$ as origin, so that $u$ is locally modelled at node $n$ by a polynomial:

$$
u^{(n)}=\sum_{r \in \mathcal{R}^{(n)}} a_{r}^{(n)} p_{r}^{(n)}
$$

where the $a_{r}^{(n)}$ are real coefficients.

We assume the families $\left(\Omega^{(n)}\right)_{n \in \mathcal{N}}$ and $\left(\varphi^{(n)}\right)_{n \in \mathcal{N}}$ to fullfil the Partition of Unity conditions i. e.:

$$
\begin{gathered}
\Omega \subset \bigcup_{n \in \mathcal{N}} \Omega^{(n)} \\
\forall \mathbf{x} \in \Omega \quad \sum_{n \in \mathcal{N}} \varphi^{(n)}(\mathbf{x})=1
\end{gathered}
$$

The global representation is then constructed by blending the $u^{(n)}$ with the PU-functions:

$$
u=\sum_{n \in \mathcal{N}} \varphi^{(n)} u^{(n)}
$$

Unlike more conventional FEMs, PUFEMs allow a patch to overlap its neighbours. For the sake of computational efficiency, our nodes are distributed over a regular rectangular 
array with an inter-node spacing $h_{i}$ along the $i^{\text {th }}$ coordinate axis $(i=1, \ldots, d)$. Each patch $\Omega^{(n)}$ is an $h_{1} \times \ldots \times h_{d}$ cuboid centered on $\mathbf{c}^{(n)}$. This configuration is illustrated on Fig. 1.a. The $\varphi^{(n)}$ are separable piecewise polynomials, equal to 1 at $\mathbf{c}^{(n)}$ and decreasing with the distance to $\mathbf{c}^{(n)}$ (cf. Fig. 1.b). Notations: in the following, $u_{i}$ denotes the $i^{t h}$ component of

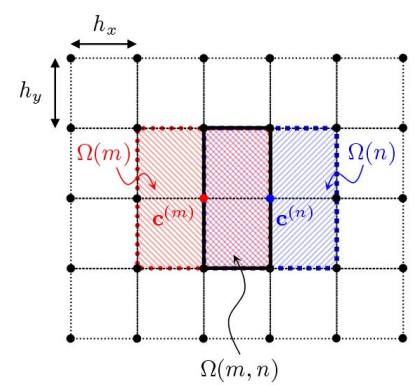

(a) Nodes and patches

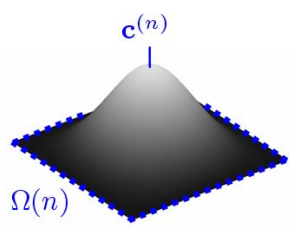

(b) PU-function
Fig. 1. Example of Partition of Unity configuration in 2D.

the displacement field $\mathbf{u}$ and $a_{i, r}^{(n)}$ the coefficients representing $u_{i} ; \mathbf{a}^{(n)}$ is the vector obtained by piling up the $a_{i, r}^{(n)}$ at node $n$ and $\mathbf{a}$ by piling up the $\mathbf{a}^{(n)}$. Similarly for a second field $\mathbf{v}$, we adopt the notations $v_{i}, b_{i, r}^{(n)}, \mathbf{b}^{(n)}$ and $\mathbf{b}$.

According to (1) and (4), $\mathbf{u}$ is as regular as the PUfunctions per se. However, we want to impose a controllable, "long range" regularization, or rather, globality. To this end, we introduce the notion of Sobolev non-conformity between two neighbouring nodes $m$ and $n$ through the energy:

$\mathcal{S}_{\kappa}^{(m, n)}(\mathbf{u})=\sum_{|\boldsymbol{\alpha}| \leqslant \kappa} \int_{\Omega^{(m, n)}} \varphi^{(m)} \varphi^{(n)}\left\|D^{\boldsymbol{\alpha}} \mathbf{u}^{(m)}-D^{\boldsymbol{\alpha}} \mathbf{u}^{(n)}\right\|^{2}$

where $\boldsymbol{\alpha}=\left(\alpha_{1}, \alpha_{2}, \ldots, \alpha_{k}\right)$ and $D^{\boldsymbol{\alpha}}$ is the partial derivative operator in the standard multi-index notations. This local energy has an intuitive interpretation: it penalizes the global field $\mathbf{u}$ if its local representations at nodes $m$ and $n$ and their derivatives up to order $\kappa$ differ in the overlapping region $\Omega^{(m, n)}$. The global conformity energy is then defined by:

$$
\mathcal{S}_{\kappa}(\mathbf{u})=\sum_{n \in \mathcal{N}} \sum_{m \in \mathcal{V}(n)} \mathcal{S}_{\kappa}^{(m, n)}(\mathbf{u})
$$

where $\mathcal{V}^{(n)}$ is the set of neighbours of node $n$ in 4-connexity. This inter-node conformity constraint is a key feature of our method. This energy is zero when all the local representations are equal, i.e. when $u$ is globally polynomial. Thus, in the case of local affine bases, global translation, rotation, scaling and shearing are not penalized.

\section{ROBUST REGISTRATION FORMULATION}

A reference image $R$ and a template $T$ are registered by minimizing the mismatch energy:

$$
\mathcal{M}(\mathbf{u})=\int_{\Omega} \rho(T \circ(\mathbf{i d}+\mathbf{u})-R)
$$

w.r.t $\mathbf{u}$. $\rho$ is a convex M-estimator in the form $\rho(s)=\Psi\left(s^{2}\right)$, which has the effect of reducing the influence of outliers (see $[8,9,10])$. For example, we choose $\Psi\left(s^{2}\right)=\sqrt{s^{2}+\epsilon^{2}}$. This energy is difficult to minimize as such and we need to remove the nonlinearity due to $T$ and $\rho$.

We first decompose the optimatization into optical flow steps. Considering one iteration, let $\mathbf{u}$ be the current unknown, $\mathbf{v}$ the solution of the previous iteration, and $\delta \mathbf{u}$ the unknown increment so that $\mathbf{u}=\mathbf{v}+\delta \mathbf{u}$. In order to remove the nonlinearity in the argument of $\rho$ in (6), we use a first order Taylor expansion of $\mathrm{T}$ :

$$
T \circ(\mathbf{i d}+\mathbf{u}) \approx T \circ(\mathbf{i d}+\mathbf{v})+\nabla T \circ(\mathbf{i d}+\mathbf{v}) \cdot \delta \mathbf{u}
$$

The framework presented in section 2 enables us to derive a simple scheme by approximating the problem by a set of independent subproblems, each confined to a node. By expanding $\delta \mathbf{u}$ as in (4) and using property (3), $\mathcal{M}(\mathbf{v}+\delta \mathbf{u})$ is approximated by:

$\int_{\Omega} \rho\left(\sum_{n \in \mathcal{N}} \varphi^{(n)}[\underbrace{T \circ(\mathbf{i d}+\mathbf{v})-R+\nabla T \circ(\mathbf{i d}+\mathbf{v}) \cdot \delta \mathbf{u}^{(n)}}_{\hat{=} \xi^{(n)}}]\right)$

$\rho$ being convex, we can apply Jensen's inequality [11] to get:

$$
\int_{\Omega} \rho\left(\sum_{n \in \mathcal{N}} \varphi^{(n)} \xi^{(n)}\right) \leqslant \sum_{n \in \mathcal{N}} \underbrace{\int_{\Omega^{(n)}} \varphi^{(n)} \rho\left(\xi^{(n)}\right)}_{\hat{=} \widetilde{\mathcal{M}}_{\mathbf{v}}^{(n)}\left(\delta \mathbf{u}^{(n)}\right)}
$$

This provides an upper bound for $\mathcal{M}(\mathbf{v}+\delta \mathbf{u})$ which we minimize instead: if all the local energies $\widetilde{\mathcal{M}}_{\mathbf{v}}^{(n)}\left(\delta \mathbf{u}^{(n)}\right)$ can be made small, then we are sure that $\mathcal{M}(\mathbf{v}+\delta \mathbf{u})$ will be small. This upper bound is interesting because it removes the dependencies between the nodes.

However the nonlinearity due to $\rho$ still remains. It can be shown that each $\widetilde{\mathcal{M}}_{\mathbf{v}}^{(n)}\left(\delta \mathbf{u}^{(n)}\right)$ can be approximated by a quadratic form of the $\delta a_{i, r}^{(n)}$ :

$\widetilde{\mathcal{M}}_{\mathbf{v}}^{(n)}\left(\delta \mathbf{u}^{(n)}\right) \approx\left(\delta \mathbf{a}^{(n)}\right)^{T} \mathbf{M}^{(n)} \delta \mathbf{a}^{(n)}+2\left(\mathbf{g}^{(n)}\right)^{T} \cdot \delta \mathbf{a}^{(n)}+C$

where $\mathrm{C}$ is a constant. $\mathbf{M}^{(n)}$ is a positive semidefinite, symmetric matrix and $\mathbf{g}^{(n)}$ a vector; their entries are defined by:

$$
\begin{gathered}
m_{(i, r),(j, s)}^{(n)}=\int_{\Omega^{(n)}} \varphi^{(n)} \Psi^{\prime}\left(\left(T_{\mathbf{v}}-R\right)^{2}\right) \partial_{i} T_{\mathbf{v}} \partial_{j} T_{\mathbf{v}} p_{r}^{(n)} p_{s}^{(n)} \\
g_{i, r}^{(n)}=\int_{\Omega^{(n)}} \varphi^{(n)} \Psi^{\prime}\left(\left(T_{\mathbf{v}}-R\right)^{2}\right)\left(T_{\mathbf{v}}-R\right) \partial_{i} T_{\mathbf{v}} p_{r}^{(n)}
\end{gathered}
$$


where $T_{\mathbf{v}} \widehat{=} T \circ(\mathbf{i d}+\mathbf{v})$ and $\partial_{i} T_{\mathbf{v}} \widehat{=} \partial_{i} T \circ(\mathbf{i d}+\mathbf{v})$. The above inequality and the latter quadratic form are generalizations of the results established in [6].

Thus, adding the inter-node conformity constraint (5), the total energy we consider at each iteration is :

$$
\begin{aligned}
\widetilde{\mathcal{E}}(\mathbf{a}) & =\sum_{n \in \mathcal{N}}\left(\mathbf{a}^{(n)}-\mathbf{b}^{(n)}\right)^{T} \mathbf{M}^{(n)}\left(\mathbf{a}^{(n)}-\mathbf{b}^{(n)}\right) \\
& +2 \sum_{n \in \mathcal{N}}\left(\mathbf{g}^{(n)}\right)^{T} \cdot\left(\mathbf{a}^{(n)}-\mathbf{b}^{(n)}\right) \\
& +\gamma \mathcal{S}_{\kappa}(\mathbf{a})
\end{aligned}
$$

with $\gamma$ being a parameter that controls the globality of $\mathbf{u}$. For example, if all the local representations are affine, then increasing $\gamma$ forces $\mathbf{u}$ towards a global affine displacement field.

\section{MINIMIZATION}

The conformity term $\mathcal{S}_{\kappa}(\mathbf{a})$ is a quadratic function of the coefficients $a_{i, r}^{(n)}$, and hence so is $\widetilde{\mathcal{E}}(\mathbf{a})$. Minimizing (7) is then equivalent to solving a linear system of size $\sum_{n} \operatorname{Card} \mathcal{R}^{(n)}$. This system is sparse since:

- the approximated matching term (i.e. the upper bound derived above) does not relate coefficients from different nodes, - the conformity (5) only relates nearest neighbour nodes.

We use conjugate gradient descent which is well-suited for solving sparse linear systems [12].

The scheme we have just described is integrated in a hierarchical environment in order to avoid local minima. We define a dyadic pyramid of PU-configurations. Once the solution has been computed at one level, it is projected on the basis of the next finer level to provide an initialization (see details in [6]). This global-to-local strategy is coupled with a multiscale approach: starting with a low-resolution image (high scale) at the coarsest level, we decrease the scale as the node array is refined.

\section{RESULTS}

We first show results on a pair of synthetic binary images to demonstrate how the globality can be controlled through the parameter $\gamma$ (cf. Fig. 2). The local bases are chosen affine. The transformation is globally affine when $\gamma$ is large (second image), and adapts locally as $\gamma$ decreases.

We applied our method to sequences of cardiac ultrasound $2 \mathrm{D}$ images, in order to track the endocardium of the left ventricle. To this end, we propagated initial manual segmentations by successively applying the displacement fields computed from each pair of consecutive frames. We used a 4-level pyramid starting from $5 \times 5$ nodes for the coarsest level to $33 \times 33$ nodes for the finest, the image being of size $608 \times 428$ pixels. The local bases are chosen quadratic.
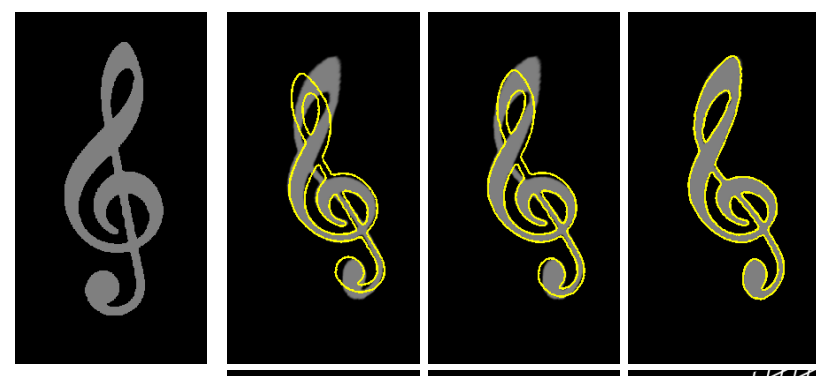

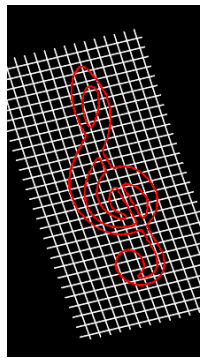

(a)

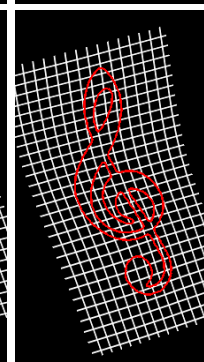

(b)

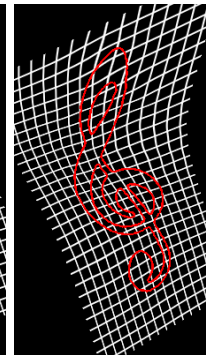

(c)
Fig. 2. The template (upper left) has been registered to the reference (a,b,c) with decreasing values of the parameter $\gamma$ : (a) 50 , (b) 5 and (c) 0.1 . The first line shows the template's contours superimposed on the reference image. The second line displays the deformations of an initially regular grid.

Fig. 3 displays results on a long-axis view sequence. Notice that the valve has an out-of-plane motion between the last two frames, but does not disturb the propagation. Fig. 4 displays results on a more difficult case: the sequence is lowcontrasted and exhibits out-of-plane motion.

\section{SUMMARY AND CONCLUSION}

We have proposed a generalization of the hierarchical Partition of Unity Finite Element Method presented in [6] to solve a variational approach to image registration. This new method offers several features to improve the robustness to outliers: the introduction of an M-estimator in the matching term is a well-known technique and can be easily integrated in the optimization scheme; the global-to-local strategy and the internode conformity constraint enable us to control the globality of the field, as opposed to existing parametric methods. In order to demonstrate the performance of our algorithm, we have applied our method to a sequence of cardiac ultrasound images to track the myocardium of the left ventricle.

In future works, we shall consider cases where abrupt spatial variations in the displacement field occur. The globality constraint may prevent the algorithm from correctly capturing the motion field. To deal with this issue, we shall introduce M-estimator techniques in the conformity penalty term. 

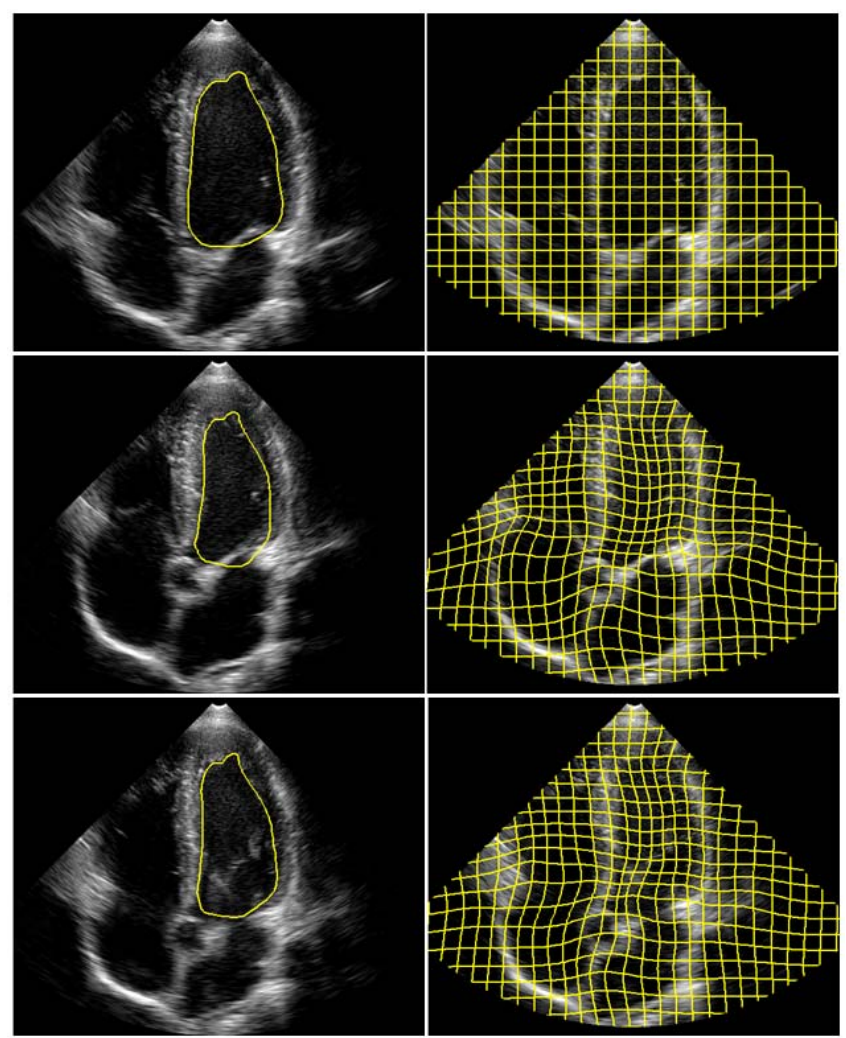

Fig. 3. Propagation of a segmentation of the left ventricle's endocardium in a long-axis view sequence. The initial contour was manually drawn (top left image). The second column shows the propagation of a grid, in order to vizualize the deformation field.

\section{REFERENCES}

[1] J.M. Fornefett, K. Rohr, and H. S. Stiehl, "Elastic registration of medical images using radial basis functions with compact support," in Proc. 1999 Conference on Computer Vision and Pattern Recognition, June 1999, pp. 402-409.

[2] D. Suter, "Motion estimation and vector splines," in Proc. 1994 Conference on Computer Vision and Pattern Recognition. 1994, pp. 939-942, IEEE Computer Society Press.

[3] B.C. Vemuri, S. Huang, S. Sahni, C.M. Leonard, C. Mohr, and R. Gilmore, "An efficient motion estimator with application to medical image registration," Medical Image Analysis, vol. 2, no. 1, pp. 79-98, September 1998.

[4] J. Kybic and M. Unser, "Fast parametric elastic image registration," IEEE Transactions on Image Processing, vol. 12, no. 11, pp. 1427-1442, November 2003.

[5] M. Ferrant, S.K. Warfield, C.R.G. Guttmann, R.V. Mulkern, F.A. Jolesz, and R. Kikinis, "3d image matching using a finite element based elastic deformation model," in Proc. 1999 MICCAI. 1999, pp. 202-209, Springer-Verlag London.

[6] S. Makram-Ebeid and O. Somphone, "Non-rigid image registration using a hierarchical partition of unity finite element

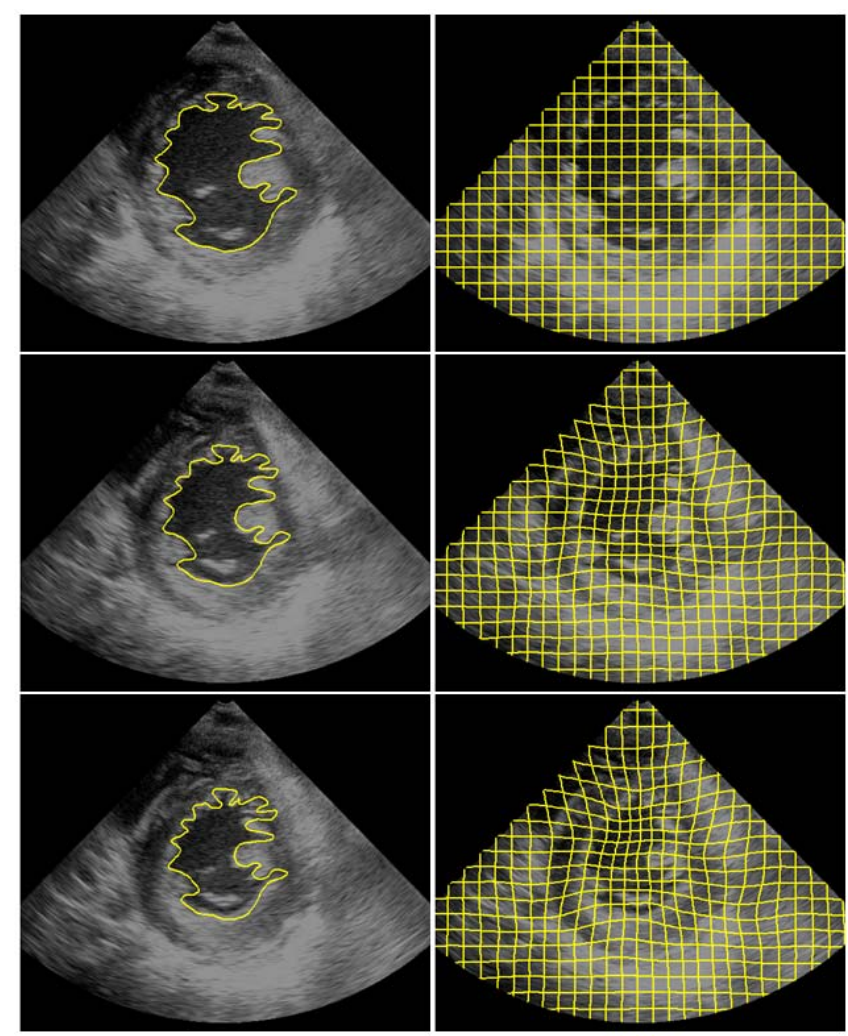

Fig. 4. Propagation of a segmentation of the left ventricle's endocardium in a short-axis view sequence.

method," in Proc. 2007 International Conference on Computer Vision, October 2007.

[7] J. M. Melenk and I. Babŭska, "The partition of unity finite element method: Basic theory and applications," Computer Methods in Applied Mechanical Engineering, vol. 139, pp. 289314, 1996.

[8] A. Bab Hadiashar and D. Suter, "Robust optic flow computation," International Journal of Computer Vision, vol. 29, no. 1, pp. 59-77, August 1998.

[9] M.J. Black and P. Anandan, "Robust dynamic motion estimation over time," in Proc. 1991 Conference on Computer Vision and Pattern Recognition, June 1991, pp. 296-302.

[10] T. Brox, A. Bruhn, N. Papenberg, and J. Weickert, "High accuracy optical flow estimation based on a theory for warping," in Proc. 2004 European Conference on Computer Vision. May 2004, pp. 25-36, Springer-Verlag Berlin.

[11] J.L.W.V. Jensen, "Sur les fonctions convexes et les inégalités entre les valeurs moyennes," Acta Mathematica, vol. 30, no. 1, pp. 175-193, December 1906.

[12] J.R. Shewchuk, "An introduction to the conjugate gradient method without the agonizing pain," Tech. Rep., Carnegie Mellon University, Pittsburgh, PA, USA, August 1994. 